\title{
Resistência à tração de coroas metálicas cimentadas com cimentos provisórios
}

\author{
Jonas Alves de OLIVEIRA'; Lara Pepita de Souza OLIVEIRA²; Juliana
} Rodrigues Paes BARRETO3; Soraia Sena de Moura QUEIROGA4

1 - Doutor em Clínica Odontológica (Prótese Dentária) - FOP/UNICAMP, Mestre em Odontologia (Reabilitação Oral) - FOB/USP, Prof. Adjunto do curso de Odontologia - ESA/UEA; 2 - Mestranda em Odontologia (Reabilitação Oral) - FAO/UFAM. Graduada em Odontologia - ESA/UEA; 3 - Mestre em Ciências Odontológicas (Materiais Restauradores) - FAO/UFAM. Graduada em Odontologia - ESA/UEA; 4 - Graduada em Odontologia - ESA/UEA.

\section{Resumo}

A cimentação provisória contribui na reabilitação estética e funcional, enquanto espera-se pela cimentação do material definitivo, sendo fundamental a utilização de cimentos com boas propriedades biomecânicas, como a resistência friccional, evitando seu deslocamento e infiltração. Objetivo: Este estudo in vitro avaliou a resistência friccional por tração na cimentação de coroas metálicas fundidas com 3 agentes cimentantes provisórios, após 7 dias e 21 dias. Material e método: foram selecionados 24 dentes humanos (terceiros molares), hígidos ou com pequenas lesões cariosas, que foram preparados conforme Técnica da Silhueta e enviados para confecção das coroas metálicas em $\mathrm{Ni}-\mathrm{Cr}$. Os corpos-de-prova foram divididos em: Grupo I: Cimento de hidróxido de cálcio (Dycal); Grupo II: Cimento de óxido de zinco e eugenol (Temp-Bond) e Grupo III: Cimento de óxido de zinco sem eugenol (Temp- Bond NE). A força de tração dos corpos-de-prova foi mensurada em uma Máquina Universal de Ensaios (Instron). A análise estatística dos dados foi realizada pelo teste ANOVA e teste de Bonferrori $(\mathrm{p}<0,05)$. Resultados: Ambos Dycal $(9,92 \mathrm{kgF})$ e Temp-Bond $\mathrm{NE}(9,52 \mathrm{kgF})$ apresentaram os maiores valores, com diferença estatística para o Temp-Bond (5,96 kgF). Conclusões: Dentre os agentes cimentantes avaliados, os sem eugenol em sua composição (Temp-Bond NE e Dycal) apresentaram melhor desempenho no quesito retenção friccional, quando comparados ao cimento com eugenol, e o tempo influenciou significativamente aumentando os valores de resistência.

PALAVRAS-CHAVE: Cimentos provisórios; Tração; Coroas metálicas. 


\section{Introdução}

$\mathrm{Na}$ busca pelo aprimoramento das diferentes formas de reabilitar a mastigação, fonação e promover estética, recuperando a saúde bucal, a Odontologia Restauradora tem evoluído, aperfeiçoando constantemente os materiais restauradores estéticos e seus mecanismos de adesão, de modo a garantir sucesso no tratamento ${ }^{1,2}$.

As restaurações indiretas, classificadas em próteses fixas, coroas totais, onlays, inlays e facetas, constituem uma opção ideal, especialmente para dentes com severa perda estrutural ${ }^{3}$, em virtude da maior resistência e garantia de longevidade. Para indicá-las, deve-se realizar um adequado planejamento, seleção de materiais apropriados, confecção de um bom preparo biomecânico e uma restauração provisória ${ }^{4}$. Tanto as restaurações finais quanto as provisórias são cimentadas, o que implica em questões biológicas, mecânicas e de interação entre os materiais, bem como na adesividade da cimentação final.

O cimento dentário atua unindo o material restaurador ao preparo do dente (ou pilar do implante) ${ }^{5}$. Como agente provisório, ele pode atuar como isolante térmico, elétrico e químico para o elemento em questão ${ }^{4,6,7}$, e também contribuir biologicamente com dentes vitais, seja por analgesia (eugenol) ou reparo pulpar (hidróxido de cálcio). O agente cimentante ideal deve ser biocompatível, com baixo potencial alérgico e possuir baixa interação com os tecidos e fluidos orais ${ }^{8}$, podendo apresentar diferentes mecanismos de união: friccional, adesão micromecânica e adesão química?.

Este material também necessita fornecer adequada resistência à tração, de modo que a restauração provisória permaneça adequadamente adaptada, em função mastigatória e, ainda, ser de fácil remoção e limpeza, quando isto for necessário, não sendo muito retentivo com o tempo, por ser um material provisório $^{10}$. O risco de fenda marginal entre o término do preparo e a restauração indireta deve ser minimizado ${ }^{5}$, o que é desafiador, 
pois sempre existirá uma linha delgada de cimento exposta aos fluidos bucais capaz de sofrer solubilização, podendo causar infiltração e hipersensibilidade ${ }^{7}$.

Para Lawson et al. ${ }^{11}$ (2006), há uma relação significativa entre a resistência flexural do cimento e a retenção da restauração provisória. Esta resistência vai ser influenciada pelo tipo de cimento, adaptação marginal da restauração e resistência à solubilidade. Ademais, deve-se atentar para uma forma de aplicação que garanta maior retenção e adaptação, preferencialmente nas paredes axiais e no terço cervical ${ }^{12}$.

Cuidados adicionais devem existir durante a seleção do cimento provisório. O eugenol, por exemplo, deve ser evitado, pois compromete a polimerização dos monômeros, o que prejudica a cimentação final, caso seja usado um cimento resinoso na cimentação definitiva ${ }^{13}$. Os cimentos à base de óxido de zinco e eugenol, quando comparados, in vitro, aos livres de eugenol, apresentam maior infiltração marginal ${ }^{5}$, retenção significativamente inferior ${ }^{14}$ e alta espessura ${ }^{15}$. Quanto ao Hidróxido de Cálcio, alguns estudos atribuem excelentes resultados quanto à resistência à tração ${ }^{10}$.

Para alcançar uma retenção adequada na coroa final, seja ela metalocerâmica ou total cerâmica, espera-se a obtenção do mecanismo de fixação dos cimentos, caracterizado pelo intercruzamento mecânico e ligação química ${ }^{16}$, assim, deve-se avaliar a melhor correlação de cimentos provisórios e definitivos no tratamento escolhido.

Diversos estudos avaliam materiais para fixação de coroas, porém, resultados conflitantes e a grande quantidade de produtos lançados periodicamente, dificulta a seleção do material por parte do cirurgião-dentista ${ }^{17}$. Assim, faz-se necessária uma análise constante destes materiais, garantindo segurança aos pacientes e profissionais. 
Os estudos científicos comparativos, que testam diferentes materiais indicados para uma mesma finalidade e em diferentes períodos de armazenagem, servem para avaliar a sua longevidade; isso permite ao cirurgião-dentista uma maior confiabilidade para selecionar o material ideal.

Posto isso, o presente estudo in vitro objetivou avaliar a resistência friccional, por tração, de coroas metálicas cimentadas com três diferentes cimentos provisórios: a base de hidróxido de cálcio, óxido de zinco com eugenol e óxido de zinco sem eugenol, após 7 dias e 21 dias, partindo-se da hipótese de que o cimento temporário a base de óxido de zinco e eugenol possui inferior resistência friccional, quando comparado a outros cimentos provisórios, e que o fator tempo possui influência nos valores de resistência.

\section{Material e método}

Este estudo foi desenvolvido na Escola de Ciências da Saúde da Universidade do Estado do Amazonas (ESA-UEA), após aprovação do Comitê de Ética em Pesquisa, de número 075/07-CEP/ESA. Foram utilizados 24 terceiros molares humanos recém-extraídos, hígidos ou com pequenas lesões cariosas, com coroas semelhantes em forma e dimensão. Os dentes foram limpos com ultrassom e mantidos em água à temperatura ambiente, durante o período do estudo. Os dentes foram posicionados e fixados em tubos cilíndricos de resina epóxi com 30,0 mm de altura, diâmetro de 20,0 mm.

Para que os dentes fossem incluídos no seu longo eixo, foi utilizado um delineador, e o posicionamento foi estabelecido no limite de 3,0 mm apical à junção cemento-esmalte com preenchimento de resina acrílica Autopolimerizável, representando a distância da inserção conjuntiva ${ }^{16,18}$. Os preparos para coroa total metalocerâmica foram realizados com instrumento cortante rotatório diamantado em alta rotação, sob refrigeração, para evitar o superaquecimento e fraturas do dente. Foi utilizada a Técnica da Silhueta ${ }^{4}$, com desgaste de 1,6 mm e término cervical em chanfrado. 
Os padrões foram obtidos com cera para incrustações e confeccionados uma alça com fio de cera n² 2, na superfície oclusal, para auxiliar na tração. As coroas enceradas foram encaminhadas de 8 em 8 para fundição. A inclusão dos padrões foi feita em anel de silicone com revestimento para incrustações, segundo as recomendações dos fabricantes. A liga de níquel-cromo foi utilizada para fundição e obtenção das coroas metálicas. As coroas foram ajustadas para detectar áreas ou pontos de atrito que impedissem seu assentamento completo nos respectivos preparos. Foi utilizado o líquido evidenciador de contato, aplicado com auxílio de um pincel pelo de marta $\mathrm{n}^{\circ} 00$ nas paredes internas das fundições. As áreas de atrito e bolhas positivas foram removidas com broca diamantada 1014 (KG Sorensen, Rio de Janeiro, RJ, Brasil), em alta rotação sob-refrigeração constante. Este procedimento foi repetido até que uma película uniforme de evidenciador fosse observada. A verificação da adaptação foi realizada com auxílio de uma sonda exploradora nº 05 e com lupa de quatro aumentos ${ }^{18}$.

Para a divisão das amostras, as coroas foram cimentadas aos dentes preparados com silicone fluido por condensação Xantopren (KULZER, Barra Funda, SP, Brasil), removidos os excessos e mantidos sob tensão em uma fresadora (Fresadora 1000N - BIOART, São Carlos, SP, Brasil) com carga de 2,0 kg durante 5 min. As películas de silicone obtidas do preparo, compatíveis com a espessura de película de cimentação, foram pesadas em balança analítica de precisão, obtendo-se valores para cada amostra. Uma vez que os dentes tinham forma e tamanho semelhantes, a divisão em grupos foi feita pela espessura de película, de modo que a média do peso entre os grupos foi semelhante (Grupo I- 14,8 mg, +/- 4,3; Grupo II- 14,1 mg, +/- 4,4; Grupo III- 13,8 mg, +/- 4,3).

Os cimentos provisórios testados foram Cimento de Hidróxido de Cálcio (Dycal), Cimento de Óxido de Zinco com Eugenol (Temp-Bond) e Cimento de Óxido de Zinco sem Eugenol (TempBond NE). Deste modo, os grupos $(\mathrm{n}=8)$ se dividiram em: 
GRUPO I - Cimentação de coroas metálicas com Cimento de Hidróxido de Cálcio (Dycal, Dentsply Ltda, Petrópolis, RJ);

GRUPO II - Cimentação de coroas metálicas com Cimento de Óxido de Zinco com Eugenol (Temp-Bond, Keer Corporation, Orange, CA);

GRUPO III - Cimentação de coroas metálicas com Cimento de Óxido de Zinco sem Eugenol (Temp-Bond NE, Keer Corporation, Orange, CA).

Antes da cimentação, todos os preparos foram limpos com pasta de pedra-pomes e água, com auxílio de escova tipo Robson, montada em contra ângulo em baixa velocidade, durante 10 segundos. Posteriormente, os dentes foram lavados em água corrente durante 10 segundos e secos com jatos de ar comprimido, livre de óleo.

As coroas metálicas, então, foram submetidas ao jateamento com óxido de alumínio $50 \mu \mathrm{m}$, depois, lavadas com água e secas com jato de ar. Cada cimento foi manipulado segundo as recomendações do fabricante (Figura 1).

Para a cimentação, foi utilizado um delineador (Figura 2), de forma que as coroas fossem cimentadas ao longo eixo do dente sob uma carga de $2,0 \mathrm{~kg}$ durante $5 \mathrm{~min}$. Para a manutenção do peso, foi confeccionada uma base de resina acrílica (Figura 3).

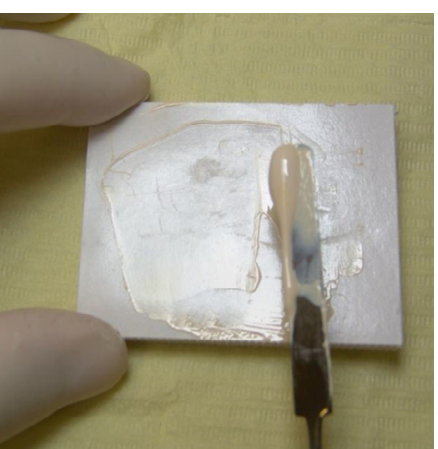

FIGURA 1 - Manipulação do cimento Fonte: Do autor (2020).

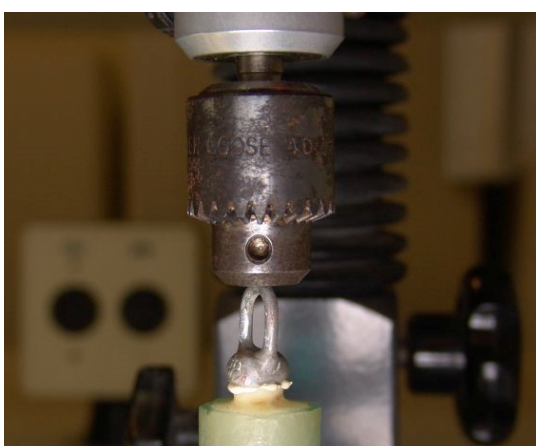

FIGURA 2 Corpo-de-prova posicionado no delineador

Fonte: Do autor (2020).

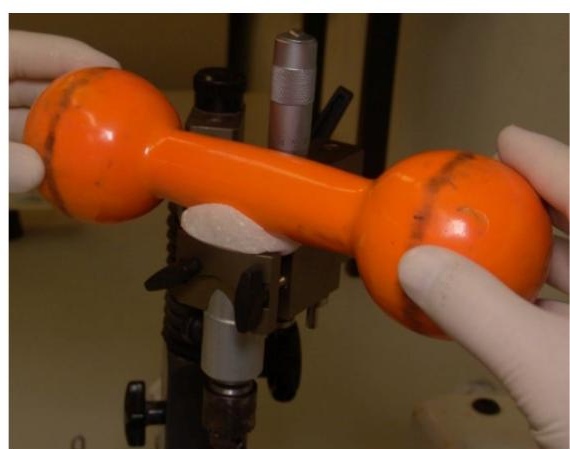

FIGURA $3 \cdot$ Peso de 2,0kg posicionado sobre o delineador

Fonte: Do autor (2020) 
Os excessos do cimento foram retirados com uma sonda exploradora $\mathrm{n}^{\circ} 5$, após a cura dos mesmos, as coroas foram armazenadas durante um período de 7 dias em água e então submetidas aos testes de tração em Máquina Universal de Ensaios (Instron , São José dos Pinhais - PR, Brasil $)^{16}$, com célula de carga de capacidade de 0 a $50 \mathrm{~kg}$ e velocidade de $0,5 \mathrm{~mm} / \mathrm{min}$.

Depois do primeiro teste e de obtidos os valores de resistência friccional após 7 dias, as coroas foram limpas com jato de óxido de alumínio $50 \mu \mathrm{m}$ e os preparos com pedra-pomes e escovas Robson. As amostras foram novamente cimentadas e armazenadas durante 21 dias em água. Após esse tempo, foi feito novo teste de retenção friccional das amostras.

Os dados foram submetidos ao teste ANOVA $(\mathrm{p}>0,05)$ e ao teste de Bonferroni ( $>00,05)$, para analisar as diferenças estatísticas entre as variáveis: material e tempo.

\section{Resultados}

Após a realização do teste de tração, obteve-se os valores de retenção friccional, em Kgf, de cada grupo após 7 e 21 dias de cimentação. Os maiores valores de retenção foram demonstrados pelo Dycal (9,92 Kgf), na cimentação de 7 dias, e pelo TempBond NE (16,43 Kgf), na cimentação de 21 dias. Já os menores valores foram observados pelo Temp-Bond tanto para cimentação de 7 dias (5,96 Kgf), como na cimentação de 21 dias $(9,39$ Kgf), conforme apresentado na Tabela 1.

TABELA 1 - Valores de média e desvio padrão da resistência à tração (Kgf) dos cimentos provisórios após 7 e 21 dias.

\begin{tabular}{lll}
\hline Cimento & Tempo & \\
\hline Dycal & $\mathbf{0 7}$ dias & 21dias \\
\hline Temp-Bond & $9,92(+/-2,31) \mathrm{Aa}$ & $12,34(+/-2,57) \mathrm{Ab}$ \\
\hline Temp-Bond NE & $5,96(+/-2,01) \mathrm{Ba}$ & $9,39(+/-2,75) \mathrm{Bb}$ \\
\hline
\end{tabular}

* Maiúsculas comparam os três cimentos entre si no mesmo período e minúsculas comparam o mesmo cimento entre os dois períodos de 7 e 21 dias $(p>0,05)$. 
Foram utilizadas as mesmas técnicas para o método de cimentação, tratamento da coroa e preparo dos dentes, estando os executores devidamente calibrados. Variando-se apenas o tipo de agente cimentante e o tempo de cimentação.

Ao analisar apenas o material utilizado, pode-se observar que houve diferença estatisticamente significante para todos os cimentos, exceto para Dycal e Temp-Bond NE na cimentação de 7 dias ( $>0,05)$. Variando-se o tempo, os três cimentos demostraram haver diferença estatisticamente significante, possuindo maiores valores na cimentação de 21 dias do que na cimentação de 7 dias ( $>>0,05)$. Ao analisar visualmente as coroas dentárias e coroas metálicas, observou-se que todas as falhas de cimentação após o teste de tração foram coesivas do cimento, não havendo nenhuma fratura dental.

\section{Discussão}

Os cimentos provisórios, de forma geral, pelo risco de solubilidade, infiltração marginal e bacteriana, devem possuir boas propriedades mecânicas, baixa solubilidade, boa adesão, e adequada resistência friccional, especialmente diante da possível necessidade de permanecerem na boca durante mais de algumas semanas ${ }^{5,14,19-21}$. Para isso, estudos são conduzidos objetivando aprimorar esses critérios ${ }^{14}$. Os Cimentos à base de óxido de zinco com eugenol e sem eugenol e o hidróxido de cálcio (Dycal) são rotineiros em cimentações provisórias, por isso foram selecionados nessa pesquisa.

Tendo em vista que as coroas provisórias apresentam maior risco de infiltração marginal, reduzindo a resistência do cimento com o tempo, o presente estudo avaliou a cimentação provisória em coroas metálicas, diminuindo a infiltração marginal, devido ao selamento marginal melhor estabelecido por esse tipo de coroa do que com a coroa provisória ${ }^{4}$, possibilitando, assim, também simular situações clínicas de casos de cimentação provisória com coroas definitivas, onde são feitos ajustes oclusais 
adicionais e de assentamento da peça, avaliação da higiene oral do paciente e estética ${ }^{10}$.

Na prática clínica, o tempo de manutenção das restaurações provisórias é outro fator importante a ser avaliado, onde é comumente estabelecido pelo profissional o prazo máximo de uma semana, todavia, em muitos casos, este tempo em boca pode prolongar, por motivos diversos, dentre eles, o retorno tardio do paciente. À vista disso, foram estabelecidos dois tempos para a análise in vitro neste estudo: 7 dias, o prazo convencional para o retorno, e 21 dias, considerando o tempo excedente.

Os valores de retenção encontrados foram maiores em 21 dias do que em 7 dias. Anusavice ${ }^{1}(2005)$ descreve que cimentos à base de água, mesmo após a presa definitiva, continuam um processo de maturação que, se em meio livre de contaminação (umidade e perda de água), torna o cimento mais resistente à dissolução, aumentando a retenção. Assim, as coroas metálicas, por sua melhor adaptação marginal do que coroas provisórias de resina acrílica, podem ter melhorado os valores de resistência à tração após 21 dias, diminuindo a solubilidade do cimento e aumentando a retenção com o tempo.

Diferentes autores mencionam a limitação do uso de cimentos a base de óxido de zinco e eugenol, devido a fatores que interferem na diminuição da sua resistência friccional, como: maior microinfiltração ${ }^{5}$, resistência flexural inferior e, portanto, menor retenção ${ }^{11}$; baixa resistên cia $^{17}$; inibição da polimerização do cimento resinoso e a alta espessura de película ${ }^{15}$. Informações estas que condizem com os resultados obtidos nesse estudo, no qual o Temp-Bond apresentou os menores valores de retenção ficcional.

O cimento de óxido de zinco sem eugenol (Temp-Bond NE), por outro lado, apresentou excelentes resultados de resistência friccional, nos dois tempos estabelecidos, esta informação confere com o estudo de Kim et al. ${ }^{6}$ (2006), feito com 30 implantes, 
no qual foi avaliado o uso de diferentes tratamentos nos "abutments” usando diferentes cimentos, onde o Temp-Bond NE apresentou maior valor de tração do que Temp-Bond.

O cimento de Hidróxido de Cálcio (Dycal), seguido do TempBond NE, apresentou maiores valores de resistência friccional no período de 7 dias, sendo ambos superiores ao Temp-Bond; dados este condizente com Ishikiriama (1984) ${ }^{19}$, no qual coroas provisórias cimentadas com cimento de Hidróxido de Cálcio (Hydro-C) apresentaram melhores resultados de retenção, se comparados ao Temp-Bond. Rego e Santiago ${ }^{10}$ (2004), por outro lado, atribuíram maior retenção ao Temp-Bond, onde as coroas cimentadas com Hydro $C$ foram mais retentivas do que TempBond $\mathrm{N}$, porém, menos retentivas que Temp-Bond; no citado estudo, porém, o período de armazenagem foi de 24 horas.

O teste de tração é um bom método para avaliar os agentes cimentantes, porém, é usualmente conduzido 24 horas após a cimentação. Porém, este intervalo de tempo é falho em mostrar mudanças ocorridas no material e na interface em períodos mais longos ${ }^{19}$. Avaliar o tempo de cimentação é importante para avaliar até que ponto o cimento mantém as propriedades mecânicas, sem desajuste ou perda da prótese. A mensuração constante das propriedades dos materiais é fundamental para permitir ao profissional selecionar aqueles que são mais apropriados e melhores para o seu caso.

No estudo, atestou-se a hipótese levantada: o cimento temporário com óxido de zinco e eugenol apresentou inferior resistência friccional, quando comparado aos outros cimentos provisórios testados, e o fator tempo influenciou nos valores de resistência. Todavia, salienta-se que a pesquisa in vitro apresenta algumas limitações, uma vez que não são considerados aspectos inerentes ao microambiente da cavidade oral, como a mastigação, participação da saliva e a dieta do indivíduo, sendo necessário considerar a contribuição de estudos clínicos que avaliem as características dos materiais, em boca e em função. Como sugestão para outros 
trabalhos deste caráter, menciona-se a inclusão de outras variáveis, como a termociclagem e o uso de coroas de resina acrílica.

\section{Conclusão}

Dentre os cimentos avaliados, em 7 e 21 dias, o cimento TempBond NE apresentou os melhores resultados, entretanto, deve-se salientar que estes são resultados mecânicos, outros fatores também são levados em consideração na escolha dos cimentos.

\section{Agradecimentos}

À Fundação de Amparo à Pesquisa do Amazonas (FAPEAM), pela concessão da bolsa de iniciação científica, pelo programa PAIC-FAPEAM-UEA.

\section{Referências}

1 - Anusavice KJ, Shen C, Rawls HR. Phillips Materiais Dentários. Rio de Janeiro: Elsevier Brasil; 2013.

2- Miura S, Fujisawa M, Komine F, Maseki T, Ogawa T, Takebe J, et al. Importance of interim restorations in the molar region. Journal of oral science. 2019; 61(2): 195-199.

3 - Peixoto RF, Aguiar CR, Jacob ES, Macedo AP, Mattos MGC, Antunes RPA. Influence of Temporary Cements on the Bond Strength of SelfAdhesive Cement to the Metal Coronal Substrate. Brazilian Dental Journal. 2015; 26(6): 637-641.

4 - Pegoraro LF, Do Valle AL, Araújo CRP, Bonfante G, Conti PCR. Prótese fixa: bases para o planejamento em reabilitação oral. São Paulo: Artes Médicas Editora; 2013.

5 - Arora SJ, Arora A, Upadhyaya V, Jain S. Comparative evaluation of marginal leakage of provisional crowns cemented with different temporary luting cements: In vitro study. The Journal of Indian Prosthodontic Society. 2016; 16(1): 42.

6- Kim Y, Yamashita J, Shotwell KL, Chong KH, Wang HL. The comparasion of provisional luting agents and abutment surface roughness on the retention of provisional implant-supported crowns. The Journal of Prosthetic Dentistry. 2006; 95(6): 450-455.

7 - Campos TND, Mori M, Henmi AT, Saito T. Infiltração marginal de agentes cimentantes em coroas metálicas fundidas. Revista Odontológica da Universidade de São Paulo. 1999; 13(4): 357-362. 
8 - Rosentiel SF, Land MF, Crispin BJ. Dental luting agents: A review of the current literature. The Journal of Prosthetic Dentistry. 1998; 80(3): 280-301.

9 - Wassel RW, Barker D, Steele JG. Crowns and other extra-coronal restorations: try-in and cementation of crowns. British Dental Journal. 2002; 193(1): 17-28.

10 - Rego MRM, Santiago LC. Retention of provisional crowns cemented with eight temporary comparative study. Journal of Applied Oral Science. 2004; 12(3): 209-212.

11 - Lawson NC, Burgess JO, Mercante D. Crown retention and flexural strength of eight provisional cements. American Association for Dental Research. 2006; 98(6): 455-460.

12 - Alabdulkader MA, Habib SR. Effect of cement application techniques on the adaptation and retention of provisional crowns. Technology and Health Care. 2018; 26(6): 1-11.

13 - Alfredo E, Souza ESD, Marchesan MA, Paulino SM, Gariba-Silva R, Sousa-Neto MD. Effect of eugenol-based endodontic cement on the adhesion of intraradicular posts. Brazilian Dental Journal. 2006; 17(2): 130-133.

14 - Lewinstein, I, Chweidan, H, Matalon, S, Pilo R.. Retention and marginal leakage of provisional crowns cemented with provisional cements enriched with chlorhexidine diacetate. The Journal of Prosthetic Dentistry. 2007; 98(5): 373-378.

15 - Yu H, Zheng M, Chen R, Cheng H. Proper Selection of Contemporary Dental Cements. OHDM. 2014; 13(1): 55-59.

16 - Consani S, Santos JG, Sobrinho LC, Sinhoreti MAC, Sousa-Neto MD. Effect of Cement Types on the Tensile Strength of Metallic Crowns Submitted to Thermocycling. Braz Dent J (2003) 14(3): 193-196.

17 - Prates LHM, Consani S., Sinhoreti MAC, Sobrinho LC. Influência de agentes cimentantes na resistência à tração de coroas totais metálicas fundidas fixadas em dentina. Pós-Grad Revista da Faculdade de Odontologia de São José dos Campos. 2000; 3(2): 90-97.

18 - Ramos Júnior L, Pandolfi RB, Valle AL. Adaptação e retenção friccional de infra-estruturas metálicas das coroas metalocerâmicas: comparação entre três técnicas usadas na confecção, antes da cimentação. Revista FOB. 1998; 6(2): 7-12.

19 - Ishikiriama A, Busato ALS, Navarro MFL, Mondelli J. Temporary cementation of acrylic resin and cast complete crowns. J Prosthet Dent. 1984; 51:637-41.

20 - Baldissara P, Comin G, Martone F, Scotti R. Comparative study of the marginal microleakage of six cements in fixed provisional crowns. J Prosthet Dent. 1998; 80: 417-422. 


\title{
Tensile strength of cemented metal crowns with provisional cements
}

\begin{abstract}
Provisional cementation contributes to aesthetic and functional rehabilitation, before definitive cementation, being essential the use of cements with good biomechanical properties, such as frictional resistance, avoiding their displacement and infiltration. Objective: This in vitro study evaluated the frictional tensile strength of cast metal crowns cemented with 3 provisional cementing agents, after 7 days and 21 days. Material and method: 24 human teeth (third molars) were selected, healthy or with small carious lesions, which were prepared according to the Silhouette Technique and sent for the manufacture of metal crowns in $\mathrm{Ni}-\mathrm{Cr}$. The specimens were divided into: Group I: Calcium hydroxide cement (Dycal); Group II: Zinc oxide and eugenol cement (Temp-Bond) and Group III: Zinc oxide cement without eugenol (Temp-Bond NE). The tensile strength of the specimens was tested on a Universal Testing Machine (Instron). The statistical analysis of the data was performed using the ANOVA test and the Bonferrori test ( $\mathrm{p}<0.05)$. Results: Both Dycal (9.92 kgF) and Temp-Bond NE (9.52 kgF) had the highest values, with a statistical difference for Temp-Bond (5.96 kgF). Conclusions: Among the cementing agents evaluated, those without eugenol in their composition (Temp-Bond NE and Dycal) showed better frictional performance retention when compared with cement with eugenol, where time influenced significantly increasing the resistance values.
\end{abstract}

KEYWORDS: Temporary cements; Traction, metal crowns.

\section{Como citar este artigo}

Oliveira JA, Oliveira LPS, Barreto JRP, Queiroga SSM. Resistência à tração de coroas metálicas cimentadas com cimentos provisórios. Rev Odontol Bras Central 2021; 30(89): 141-153. DOI: 